\title{
EEGLAB - AN OPEN SOURCE MATLAB TOOLBOX FOR ELECTROPHYSIOLOGICAL RESEARCH
}

\author{
Clemens Brunner ${ }^{1}$, Arnaud Delorme ${ }^{2}$ and Scott Makeig ${ }^{2}$ \\ ${ }^{1}$ Institute for Knowledge Discovery, Graz University of Technology, Austria \\ ${ }^{2}$ Swartz Center for Computational Neuroscience, Institute for Neural Computation, University of California \\ San Diego, CA, USA \\ clemens.brunner@tugraz.at
}

\begin{abstract}
EEGLAB is a widely used open-source MAT$L A B$ toolbox for analysis of electrophysiological data. Using EEGLAB, users can import various data formats, preprocess data (filter, resample, average, epoch), visualize data (signal browser, event-related potentials, power spectra), perform independent component analysis (ICA), use various timelfrequency analysis methods such as eventrelated spectral perturbation (ERSP) and inter-trial coherence (ITC). The extensible plug-in architecture enables third parties to contribute additional functionality such as source localization, connectivity estimation or the design of online brain-computer interfaces.
\end{abstract}

Keywords: Biosignal analysis, MATLAB, software, open source, GPL

\section{Introduction}

The EEGLAB signal processing environment, an open source software project of the Swartz Center for Computational Neuroscience (SCCN) of the University of California San Diego (UCSD), began as a set of electroencephalogram (EEG) data analysis tools for MATLAB (The Mathworks, Inc.). These tools were made publicly available by Scott Makeig in 1997, which evolved into EEGLAB, which was first released with a coherent structure and graphical user interface in 2001. More than ten years later, the EEGLAB reference paper [1] has over 2,500 citations, the opt-in EEGLAB discussion email list links over 6,500 researchers, the EEGLAB news list has over 10,000 subscribed researchers, and a recent survey of 687 research respondents has reported EEGLAB to be the software environment most widely used for electrophysiological data analysis. In addition, at least 35 EEGLAB plug-in tools have now been released by researchers from many laboratories. EEGLAB is now a de facto standard supporting a wide range of EEG and other electrophysiological research studies with contributions from many researchers.

\section{Data import and preprocessing}

EEGLAB supports importing numerous different data formats such as ASCII, MATLAB, BCI2000, Neuroscan, Biosemi, ANT EEProbe, and BrainVision. In addition, EEGLAB includes some functions from BioSig ${ }^{1}$ and Field-

${ }^{1}$ biosig. sourceforge. net

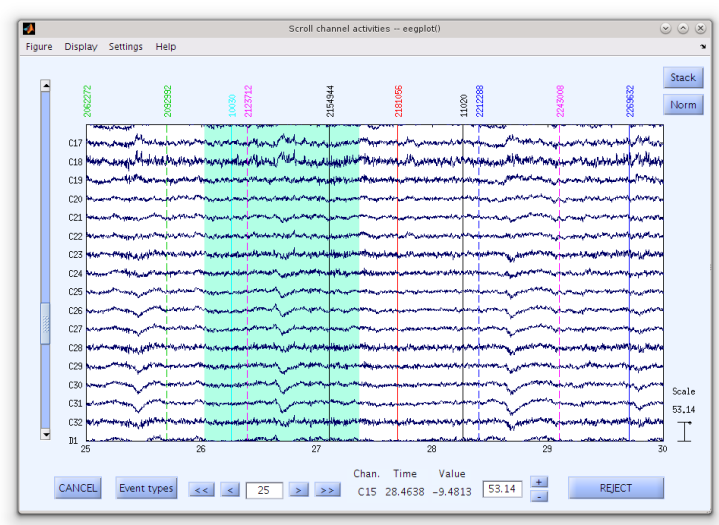

Figure 1: Signal browser showing 17 EEG channels with colored event markers, control buttons, and a selected segment (green background between 26-27.4 s).

Trip $^{2}$ that together provide support for many other data formats.

Standard preprocessing methods available in EEGLAB include filters (FIR and IIR), epoch extraction, baseline removal, resampling, and re-referencing. Furthermore, data channels can be deleted or interpolated, and artifacts can be removed using fully- or partially-automated methods (based on channels or epochs).

\section{Data visualization}

Data can be visualized in a scrollable interactive view which displays a specific number of channels in a specific time range. Figure 1 shows this channel activities window with event markers and a custom selection. This visualization is particularly useful for manual artifact rejection, where the user selects signal segments by clicking and dragging with the mouse.

\section{Independent component analysis (ICA)}

A particular strength of EEGLAB is its seamless integration of independent component analysis (ICA). ICA algorithms can be used to detect and isolate stereotypical artifacts (such as eye movements and electrocardiographic signals) mixed by volume conduction in EEG recordings. Fur-

\footnotetext{
${ }^{2}$ fieldtrip.fcdonders.nl
} 


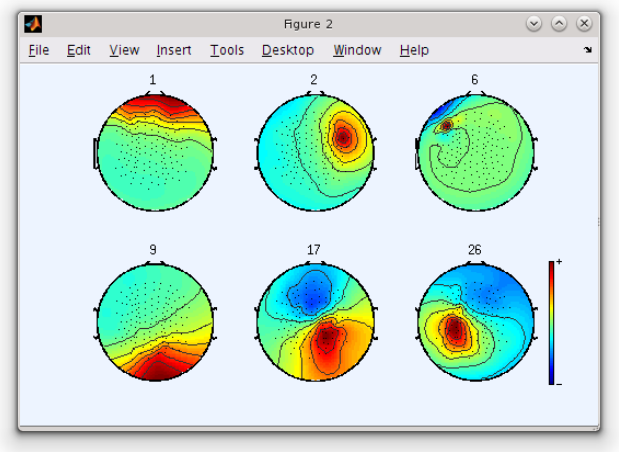

Figure 2: Scalp maps for six independent components from a 128-channel decomposition.

thermore, ICA algorithms are capable of separating biologically plausible dipolar brain sources. The default ICA algorithm available in EEGLAB is extended Infomax [2], but other algorithms including $\mathrm{JADE}^{3}$, FastICA ${ }^{4}$, and $\mathrm{AMICA}^{5}$ are also available in the core EEGLAB distribution or as plug-ins. Most visualization functions and processing tools are available for both channel and component data (for example, ERP plotting, time/frequency plots, power spectra, artifact rejection, data visualization), so it is straightforward to work with either or both signal types. Figure 2 shows scalp maps of selected independent components. Dipole source localization of such maps is possible using DIPFIT2 (included) or the $\mathrm{NFT}^{6}[3]$ toolbox.

\section{Time/frequency analysis}

EEGLAB supports time/frequency analysis measures such as (baseline or averaged) power spectrum, event-related spectral perturbation (ERSP), inter-trial coherence (ITC), and event-related cross-coherence. The SIFT $^{7}$ toolbox offers a wide range of additional connectivity measures.

ERSP visualizes event-related changes in the averaged power spectrum in a broad frequency range relative to a baseline interval. ITC measures the amount of event-related phase-locked activity as a function of time and frequency, whereas ERCOH calculates event-related coherence between two signals.

\section{User interface}

EEGLAB features a graphical user interface (GUI) useful for new users and for exploring new data. However, all functions are also accessible via the command line at two different levels: (1) so-called pop functions are the basis of all GUI functionality and are also available through EEGLAB's command history, and (2) low-level functions and data structures. MATLAB scripting is fully supported.

\footnotetext{
${ }^{3}$ bsp.teithe.gr/members/downloads/Jade

4 research.ics.aalto.fi/ica/fastica/

${ }^{5}$ sccn.ucsd.edu/ jason/amica_web.html

${ }^{6} \mathrm{sccn}$. ucsd. edu/wiki/NFT

${ }^{7}$ scen.ucsd.edu/wiki/SIFT
}

\section{Documentation and support}

EEGLAB is distributed under the GNU General Public License, the complete source code is publicly available.

EEGLAB is based on (and therefore requires) the commercial MATLAB software environment (version 7 or higher). However, all dependencies on additional MATLAB toolboxes have been removed from the core EEGLAB distribution (some advanced EEGLAB plug-ins may still require specific MATLAB toolboxes).

Most EEGLAB functions also run under Octave, an open source software environment compatible with MATLAB. However, graphical output might not be fully functional.

EEGLAB documentation is freely available on the $\mathrm{web}^{8}$, featuring a hands-on tutorial, an online workshop, a wiki, frequently asked questions (FAQ), a bug report interface, mailing lists for news and discussions, and a support email account (these email addresses can be found on the EEGLAB website).

\section{Discussion}

The functionality of EEGLAB can be extended with plugins. Currently, the community has contributed around 35 EEGLAB plug-ins, ten of which are included in the core EEGLAB distribution (for example, DIPFIT2 from FieldTrip, BioSig data import, IIRfilt, and LORETA ${ }^{9}$ ).

Future development of EEGLAB will focus on supporting, maintaining, and integrating important plug-ins. Major examples include the in-house developed toolboxes BCILAB, SIFT, MPT, NFT, and MoBILAB [3]. EEGLAB core tools will be strengthened (including improved source localization, a wider range of ICA and beamforming algorithms, and support for computations on high-performance clusters, GPUs, and the cloud). Finally, a new web infrastructure will be developed (including a new software hosting facility and a comprehensive community website featuring more extensive documentation and tutorials).

\section{Bibliography}

[1] A. Delorme and S. Makeig, "EEGLAB: an open source toolbox for analysis of single-trial EEG dynamics including independent component analysis," Journal of Neuroscience Methods, vol. 134, no. 1, pp. 9-21, 2004.

[2] A. J. Bell and T. J. Sejnowski, "An informationmaximization approach to blind separation and blind deconvolution," Neural Computation, vol. 7, no. 6, pp. 1129-1159, 1995.

[3] A. Delorme, T. Mullen, C. Kothe, Z. Akalin Acar, N. Bigdely-Shamlo, A. Vankov, and S. Makeig, "EEGLAB, SIFT, NFT, BCILAB, and ERICA: new tools for advanced EEG processing," Computational Intelligence and Neuroscience, vol. 2011, p. 130714, 2011.

\footnotetext{
${ }^{8}$ sccn.ucsd.edu/wiki/EEGLAB

${ }^{9}$ www.uzh.ch/keyinst/loreta.htm
} 